\title{
Damage monitoring in fibre reinforced mortar by combined digital image correlation and acoustic emission
}

\author{
Simon Rouchier*1 , Geneviève Foray ${ }^{2}$, Nathalie Godin ${ }^{3}$, Monika Woloszyn ${ }^{4}$, and Jean-Jacques \\ Roux $^{5}$ \\ ${ }^{1}$ CETHIL CNRS UMR5008, MATEIS CNRS UMR5510, INSA-Lyon, Université Lyon 1, F-69621 Villeurbanne, France \\ ${ }^{2}$ INSA-Lyon, MATEIS CNRS UMR5510, F-69621 Villeurbanne, France \\ ${ }^{3}$ INSA-Lyon, MATEIS CNRS UMR5510, F-69621 Villeurbanne, France \\ ${ }^{4}$ LOCIE, CNRS-UMR5271, Université de Savoie, Campus Scientifique, Savoie Technolac, 73376 Le Bourget-du-Lac Cedex, France \\ ${ }^{5}$ CETHIL, CNRS UMR5008, INSA-Lyon, Université Lyon 1, Bât. S. Carnot, 9 Rue de la Physique, F-69621 Villeurbanne, France
}

Postprint: Rouchier S., Foray G., Godin N., Woloszyn M., Roux J.-J., 2012. Damage monitoring in fibre reinforced mortar by combined digital image correlation and acoustic emission, Construction and Building Materials vol. 38, pp. 371-380. http://dx.doi.org/10.1016/j.conbuildmat.2012.07.106

\begin{abstract}
The present work aims at developing a methodology for the detection and monitoring of damage and fractures in building materials in the prospects of energetic renovation. Digital image correlation (DIC) and acoustic emission (AE) monitoring were simultaneously performed during tensile loading tests of fibre reinforced mortar samples. The full-field displacement mappings obtained by DIC revealed all ranges of cracks, from microscopic to macroscopic, and an image processing procedure was conducted as to quantify their evolution in the course of the degradation of the samples. The comparison of these measurements with the acoustic activity of the material showed a fair match in terms of quantification and localisation of damage. It is shown that after such a calibration procedure, AE monitoring can be autonomously used for the characterisation of damage and fractures at larger scales.
\end{abstract}

Keywords damage; mortar; non destructive testing; digital image correlation; acoustic emission

\section{Introduction}

The ageing of building materials and components has many repercussions on their mechanical, hygric and thermal properties: damaged materials are more permeable to moisture which may increase degradation, cause mould problems, bring health problems or form thermal bridges, sinking the overall energetic performance of the building. In order to properly estimate the overall performance of existing buildings in the prospects of energetic renovation, one must dispose of a reliable way to characterize damage and fractures in such materials. There is a high interest in extending the use of non destructive damage observation to the case of building materials, in order to help decision processes for maintenance. Moreover, because of environmental concerns, building facades tend to include multi-layered components such as outer thermal insulation, of which durability must be assessed. In such cases, damage monitoring aims at preventing the degradation of hygrothermal properties.

Imaging techniques for crack detection include X-ray radiography and tomography [34] for three-dimensional fracture observation [15] or propagation monitoring [16]. The detection of pre-existing cracks can be facilitated

*Electronic address: simon.rouchier@insa-lyon.fr; Corresponding author 
by impregnation techniques [13] allowing their automatic detection and quantification [2] by microscopic examinations. While these techniques may have a good resolution and precision, they are limited in terms of specimen size and thus not applicable on the building scale. Another possible method for such large scale applications is the recording of elastic wave velocities, which can for instance be applied to durability studies or to repair work assessments [32].

Displacement mapping techniques are applicable to damage monitoring, and consist in measuring local displacements of a sample during its deformation. Electronic speckle pattern interferometry $[14,30]$ and digital image correlation (DIC) [36] are optical non-destructive techniques and can be used for the observation of the progressive crack development, or more generally of two-dimensional strain mapping. Optical methods have also been recently extended to three-dimensional displacement and strain fields [24, 4].

A third category of damage monitoring methods includes the recording of acoustic emissions (AE). In a material under loading, elastic waves are emitted as a consequence of crack initiation and propagation. Recording and analysis of acoustic activity is of great importance in the fields of civil engineering and material sciences. Among others, it has been used for damage estimation of concrete [23, 7, 37], identification of damage mechanisms [25, $10,9,22,19]$, estimation of the fracture energy [21] or failure prediction [31, 20, 18].

Acoustic emission and other wave propagation measurement techniques are the most preferable methods for large scale damage monitoring, but the interpretation of recorded waveforms requires preliminary studies. Digital image correlation and acoustic emission recording were simultaneously performed during tensile loading tests of fibre reinforced mortar samples. The methodology aims at correcting the downsides of two techniques by combining them: the applicability of optical techniques hardly extends to field studies, while the interpretation of $\mathrm{AE}$ measurements is difficult without a view of the crack evolution. A methodology is presented, that allows interpreting acoustic signals on the basis of a previous characterization. Mortar samples were loaded by uniaxial tension while the local displacements of their surface were monitored by a camera, and the acoustic activity was recorded by sensors placed around the damaged area. By correlating the data gathered by the two techniques during this lab experiment, it is possible to interpret in field AE measurements with more clarity. The main target is therefore to calibrate the AE technique, so that it can be autonomously used for damage monitoring of building components.

\section{Methodology}

\subsection{Experimental setup}

The studied material is a commercial formulation (Lafarge, MAITE monocomposant) used for external thermal insulation composite systems. It is a Portland cement mortar including dry redispersable polymer systems with a water to dry material weight ratio of 0.16 and reinforced with $1 \%$ weight of glass fibres. The purpose of the fibers is to improve the ductility of an originally fairly brittle material. A more complete explanation of the elaboration procedure was presented in [27]. The material was cast into prismatic samples of 500 grams and dimensions $300 \times 100 \times 10 \mathrm{~mm}$, kept two days at a $90 \%$ relative humidity and 21 days at $50 \% \mathrm{RH}$. Granulometric analysis of this material has been presented by [5], along with a thorough description of its behaviour during hydration. It has already shown an ability for multi-cracking [4], and is thus expected to facilitate the progressive visualisation of crack patterns.

The prismatic samples were notched as to ensure stress concentration on a relatively small area during mechanical loading, on which the observation was focused. Tensile loading was applied along the vertical axis, using a $5 \mathrm{kN}$ force cell imposing a constant displacement speed of $1 \mathrm{~mm} / \mathrm{min}$. The tests were carried out until complete failure of the specimen. The setup of the samples and of the observation equipment is displayed on Fig. 1.

The experimental setup includes a CCD camera with a fixed 2.8 focal ratio, focused on the surroundings of the notch. Because of the shallow depth of field of the camera in this configuration, the focus is manually fixed as to prevent an automatic adjustment of the lens during the tests. The camera was positioned as to cover an observation zone of $101 \times 67 \mathrm{~mm}$ with a spatial resolution of $22 \mu \mathrm{m} /$ pixel. A non-uniform speckle pattern was applied on the specimen surface with a black paint spray as to facilitate the procedure of the correlation algorithm: this ensures that the initial gray-scale level distribution. Pictures were taken at time intervals of 5 or 10 seconds in order to allow computation of the displacement mappings during successive stages of loading. The camera 


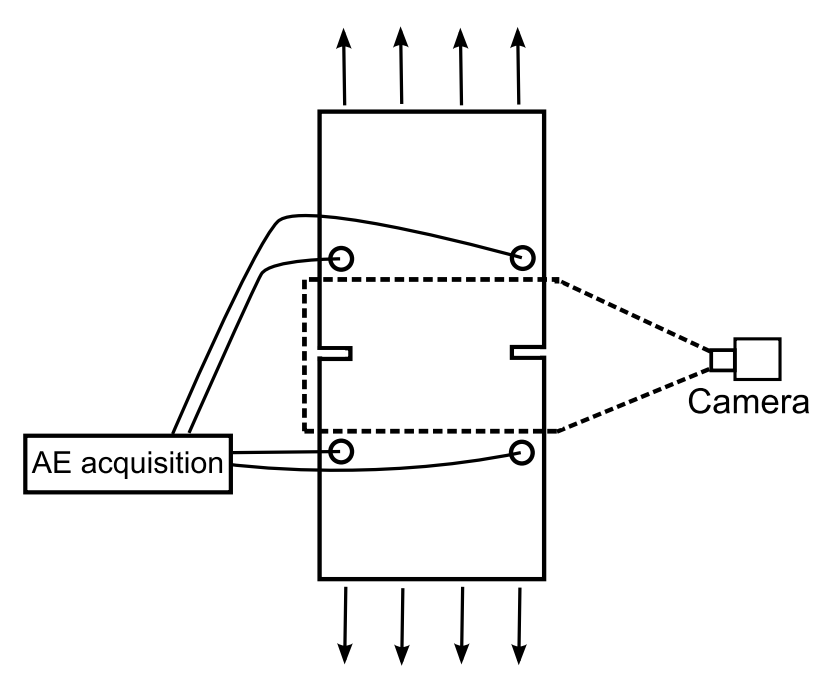

Figure 1: Experimental setup for tensile loading and damage monitoring

was used as an optical extensometer to monitor the macroscopic strain in the direction of loading. The material presents some ductility before the peak of loading [27], followed by a strain-softening behaviour during which crack propagation is slowed by the presence of fibres. This behaviour facilitated the process of progressive damage monitoring by acoustic and optical measurements.

In addition to the optical monitoring apparatus, four Micro- 80 acoustic sensors were placed around the monitored area of the specimen, continuously recording the AE activity during damage and fracture propagation. The sensors form a $80 \times 70 \mathrm{~mm}$ (height $\times$ width) area and are connected via pre-amplifiers to a MISTRAS data acquisition system. They have a diameter of $8 \mathrm{~mm}$, and are characterised by the position of their center. The settings of the $\mathrm{AE}$ recording setup are detailed below.

The methodology has been applied on a series of samples, 6 of which showed suitable for data interpretation (i.e. stress concentration and fracture occured in the observation area). Results are presented in two parts: first (Sec. 3), the ability of digital image correlation for the observation of fracture patterns is assessed. Strain mappings are calculated by the DIC algorithm and a procedure is explained for observing the evolution of crack size distributions during tensile tests. In the second part (Sec. 4), these optical measurements serve as a basis for the interpretation of AE recordings. Measurements performed on all samples were used to establish the ability of AE to quantify, locate and identifty damage and fractures.

\subsection{Digital image correlation}

Optical techniques such as DIC are non-destructive and therefore do not disturb eventual further testing on the samples. DIC provides a full map of the deformations at the surface of a specimen and allows following the fracture development without restriction of number or size of the cracks. It also presents the advantage of being easily implemented into most experimental setups, requiring no strict operating conditions or time consuming preparation, nor does it require gauges in contact with the specimen which might interfere with the experiment. The technique enables full field measurements of the local displacements of a sample's surface. It has been used for the estimation of stress intensity factors near crack tips [26, 29] or the identification of elastic properties [12] or damage laws [17], among other uses. The technique has been proven suitable for the observation of local displacements of brittle building materials such as concrete $[6,8,28]$. The principle of DIC is briefly summed up below. For a more complete view of its theory and applications, one can refer to a recent book on the subject [35].

The principle of digital image correlation is the conservation of the optical flow between two pictures of a specimen, taken at different stages of its deformation [36]: it lies on the assumption that two consecutive images contain the same total amount of each gray-scale level, and are only distinguished by their spatial distributions. 
The planar displacement $\mathbf{u}(\mathbf{x})$ at each pixel of coordinates $\mathbf{x}$ of a surface is defined as:

$$
g(\mathbf{x}+\mathbf{u})=f(\mathbf{x})
$$

where $f$ is the distribution of gray-scale level, or the texture, of a reference image, typically taken at the start of the mechanical loading, and $g$ is that of a deformed image. Eq. 1 means that the gray-scale level of a pixel of coordinates $\mathbf{x}$ on the initial image $f$ is equal to that of a pixel of coordinates $\mathbf{x}+\mathbf{u}$ on the final image $g$, i.e. that this pixel has been displaced by a vector $\mathbf{u}$. The exact displacement field $\mathbf{u}(\mathbf{x})$ can generally not be explicitely calculated without additional assumptions of regularity. In the prospects of the numerical resolution of Eq. 1, the functional $\phi$, operating on displacement fields, is defined:

$$
\phi(\mathbf{v})=\iint(g(\mathbf{v}+\mathbf{x})-f(\mathbf{x}))^{2} \mathrm{~d} \mathbf{x}
$$

where $\mathbf{v}$ is an approximation of the solution $\mathbf{u}$, and is constructed as a linear combination of functions $v_{i}$ :

$$
\mathbf{v}(\mathbf{x})=\sum N_{i}(\mathbf{x}) v_{i}
$$

where $N_{i}$ are chosen basis functions (typically bilinear functions of $\mathbf{x}$ ). The target is to determine the best possible approximation by minimizing the value of $\phi$ on all elements of the grid. Assuming a certain smoothness of the investigated displacement field, $g$ is replaced by its first-order Taylor expansion in Eq. 2, resulting in:

$$
\left[\iint(\nabla g(\mathbf{v}+\mathbf{x}) \otimes \nabla g(\mathbf{v}+\mathbf{x})):\left(N_{j} \otimes N_{i}\right) \mathrm{d} x\right] v_{i}=\iint(f(\mathbf{x})-g(\mathbf{x})) \nabla g(\mathbf{x}) N_{i}(\mathbf{x}) \mathrm{d} \mathbf{x}
$$

Since $f, g$ and $N_{i}(\mathbf{x})$ are known, Eq. 4 is a linear system of unknowns $v_{i}$, from which the approximate displacement field $\mathbf{v}$ can be obtained. Understandably, the choice of the basis functions $N_{i}(\mathbf{x})$ is important for a good approximation of the displacement field, and several options have been presented in the literature for different types of mechanical tests. In the present case, the computation of the displacements is achieved with the Icasoft software $^{1}$, which uses bilinear finite element shape functions. The displacement field is analysed by dividing the image into grid elements, each of which is assigned two displacement components in the decomposition of Eq. 3. The result of Eq. 4 is a rough estimate of the real solution, since it only computes an integer number of pixels for the displacement fields. Subpixel displacements can be determined by interpolating the displacement fields between elements of the grid with bilinear or spline functions [12].

The result of the algorithm is an approximation of the displacement field $\mathbf{u}$ of each point of the surface. While gradients of $\mathbf{u}$ define the strain fields, a local displacement jump indicates a discontinuity that appeared after the reference image was taken: in quasi-brittle materials, it translates as a crack of measurable aperture.

\subsection{Acoustic emission}

\subsubsection{Principle}

In a material under loading, elastic waves are emitted as a consequence of microstructural dislocations or crack propagation suddenly releasing stored energy. An AE hit occurs as a signal captured by a sensor exceeds a pre-set threshold value of amplitude. The result of the measurement procedure is a set of temporally separated AE hits being captured during the recording process. In the present study, the interpretation of these measurements is threefold: we investigated how AE signals can be used for damage quantification, localisation and identification.

Quantitative damage estimations consist in measuring the mechanical degradation state of a material sample on the basis of the number of recorded AE signals, or on their generation rate. Ohtsu and Watanabe [23] performed such a diagnostic procedure on concrete samples and established a relationship between the AE emission rate and damage evolution. Similar analyses were later carried out by Suzuki and Ohtsu [37], among others.

It is also possible to locate the source of a signal by placing several sensors in contact of a specimen. When the propagation speed of AE waves in the given material is known, the different arrival times of a same hit at each

\footnotetext{
${ }^{1}$ Icasoft Digital Image Correlation Software - http://icasoft.insa-lyon.fr/
} 
sensor enables the localisation. The source can be located on a 1D axis with two sensors, on a plane with three and in 3D with four. Examples of 2D or 3D crack and damage localisation include [11, 22] and generally employ more sensors than strictly necessary, for accuracy purposes. In the following, the term of AE event denotes a signal received by all sensors placed on the sample.

\subsubsection{Settings}

The propagation speed of acoustic waves in each material sample was measured prior to the tests with the lead breaking procedure: a repeatable AE wave is generated on the specimen surface by breaking pencil leads and measuring the difference in arrival times between sensors. This method also allows measuring the attenuation of the material, i.e. the amplitude decrease of a wave per unit length, and setting the timing parameters of the acquisition apparatus (peak definition time, hit definition time and hit lockout time), which are set for distinguishing signals from an uninterrupted AE stream. The average measured values of the propagation speed and attenuation coefficient are shown on Tab. 1, along with the settings of the AE recording system.

\begin{tabular}{|ll|}
\hline Preamplifier gain & $40 \mathrm{~dB}$ \\
Threshold of detection & $32 \mathrm{~dB}$ \\
Peak definition time & $50 \mu \mathrm{s}$ \\
Hit definition time & $100 \mu \mathrm{s}$ \\
Hit lockout time & $1000 \mu \mathrm{s}$ \\
Propagation speed & $2313440 \mathrm{~mm} \cdot \mathrm{s}^{-1}$ \\
Attenuation & $0.25 \mathrm{~dB} \cdot \mathrm{mm}^{-1}$ \\
\hline
\end{tabular}

Table 1: Settings of the AE acquisition system

During mechanical tests, four AE sensors were placed on the specimen as shown on Fig. 1, forming a $80 \times 70$ $\mathrm{mm}$ area. The acquisition system records the waveform of all $\mathrm{AE}$ hits received by all sensors. When a signal is captured by three or more sensors within a small enough time frame, a localisation algorithm is run as to calculate the 2-dimensional coordinates of its source. In case of a signal captured by four sensors, the calculation is repeated for all combinations of three, and the final result is the averaged value of all combinations. The following usual assumptions are made for simplifications purposes: the specimen thickness is neglected, AE propagation speed is uniform and constant (not influenced by damage). Because of these assumptions, and of the heterogeneity of the material, uncertainties are expected in the results of the localisation process.

\subsubsection{Accuracy assessment of the 2D localisation}

An accuracy test of the signal localisation algorithm was performed prior to the mechanical loading tests. First, the isotropy of the material in regards to the propagation speed was confirmed. Then, sensors were placed on a specimen surface and pencil leads were broken on a set of test points. Five lead breaks were performed on each of the five test points. Pencil breaks have a high energy release and are easily distinguishable from background noise: weaker events were filtered out, and stronger ones were localised. The results are shown on Fig. 2.

The accuracy of the localisation algorithm is estimated by comparing the exact origins of the signals (the test points) with the computed ones: the overall precision of the results is satisfactory but irregular. Acoustic waves generated in the center of the monitored area are the most accurately located. Inversely, signals originating from the sides of the monitored area are found with less precision. The material is indeed heterogeneous and the propagation speed may exhibit local variations due to variable pore and grain sizes [27]. The accuracy of the algorithm is however considered sufficient for the purpose of the study. 


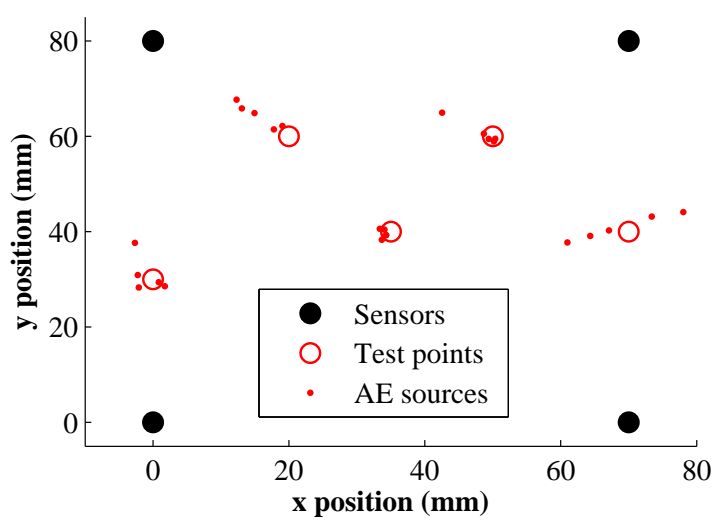

Figure 2: Accuracy testing of the localisation procedure

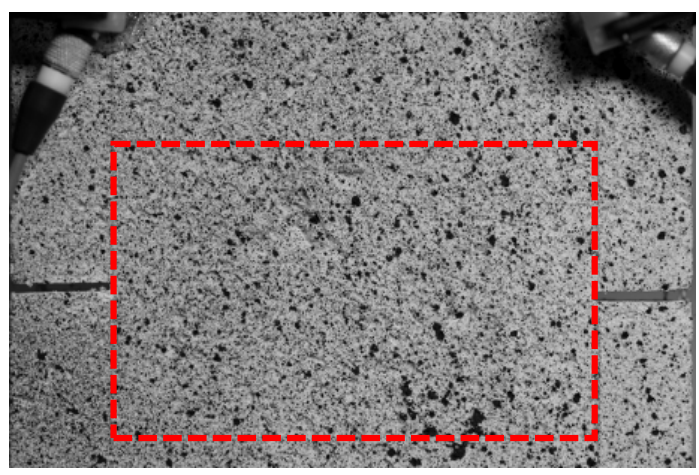

Figure 3: Original picture in 8-bit gray scale showing the input area of the DIC algorithm

\section{Imaging results}

All material samples were loaded as described above and pictures of their surfaces were taken at time intervals of 5 or 10 seconds during loading. For purposes of clarity, the procedure for extracting the crack size distributions from the optical measurements is illustrated with one of the samples (later labeled as sample 1). The results are then displayed for all samples on Fig. 6.

The correlation algorithm works by applying Eq. 4 to the reference image $f$ (taken at the beginning of the loading process) and the deformed image $g$ (taken during loading), in order to compute the displacement field over a grid on the specimen surface. Fig. 3 shows the image of the sample surface from the CCD camera shortly after crack initiation, before its input into the correlation algorithm. The calculation area of the DIC algorithm is delimited by the notches and shown by the red dotted line.

The influence of the grid size on the accuracy of the subpixel interpolation algorithm has been studied by many authors (see for instance $[3,12]$ ). With the settings of the present work, i.e. a grid composed of $8 \times 8$ pixel elements, performance estimations show a possible accuracy of $10^{-1}$ to $10^{-2}$ pixel for the computation of the displacement fields: in the present case, the displacement uncertainty is under $1 \mu \mathrm{m}$. An example of strain mapping resulting from the correlation procedure is shown on Fig. 4, on which the gray scale levels indicate the local value of the longitudinal strain in the direction of loading.

This local strain value is the gradient of displacement between adjacent pixels. Since the material is quasibrittle, locally high values indicate the presence of cracks. These cracks originate from the tips of the notches ( $y=0 \mathrm{~mm}, x=0$ or $70 \mathrm{~mm}$ ) and spread on a relatively large area of over $40 \mathrm{~mm}$ height. The present example shows the state of the sample surface shortly after the initiation of a macroscopic fracture (see Fig. 4(a)). The displayed 


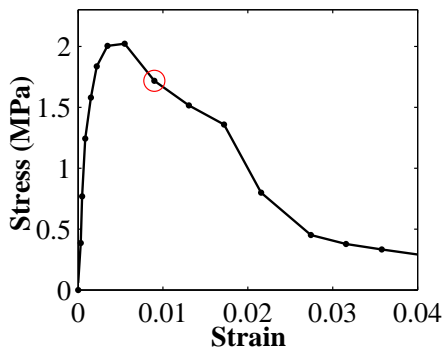

(a)

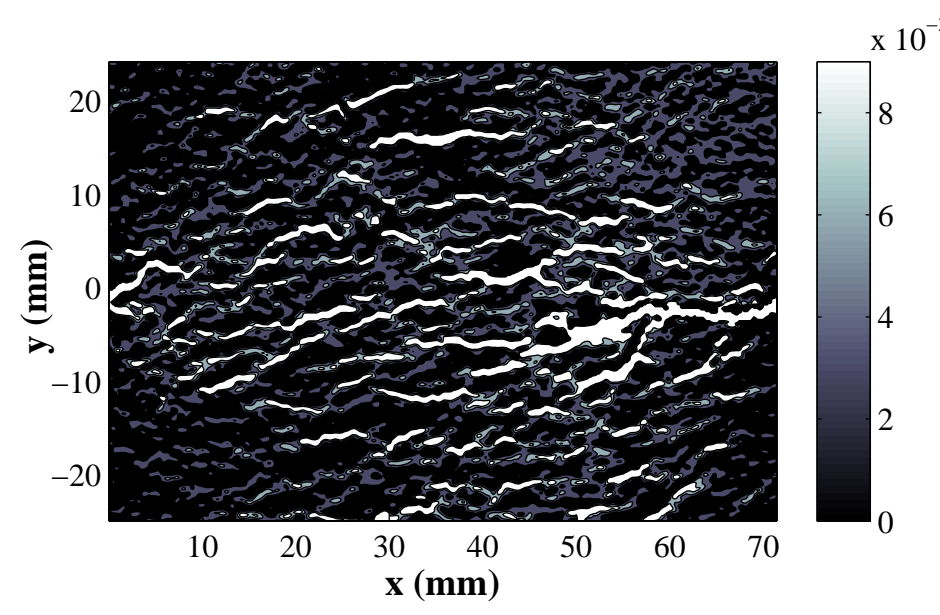

(b)

Figure 4: Example of a longitudinal strain mapping

strain mapping (Fig. 4(b)) therefore includes cracks of a wide range of apertures.

On the basis of these full field strain mappings, a methodology is proposed and implemented to measure the aperture and total length of all cracks appearing on the sample surface: successive thresholds were applied on each strain mapping, as to only display cracks exceeding certain values of aperture. Image processing of the resulting profiles allowed measuring the total developed crack length in each width interval. An example of this procedure is shown on Fig. 5.

Fig. 5(a) and 5(b) show the strain mapping 4 once it has been thresholded as to only display discontinuities of over $2 \mu \mathrm{m}$ and $5 \mu \mathrm{m}$, respectively. The appearing patterns were processed by topological skeletonisation in order to reveal cracks as tortuous linear paths: the skeleton of a shape is a thin version that is equidistant to its boundaries, and preserves its main geometrical properties, such as its length, connectivity and direction. The ImageJ software was used for this process. As an example, its applicaton to the distributions showed on Fig. 5(a) and 5(b) are respectively on Fig. 5(c) and 5(d). The resulting paths are measurable, and the substraction of the total visible crack length of Fig. 5(c) by that of Fig. 5(d) gives the amount of cracks of which aperture $w$ is in this interval. The procedure was performed on each successive strain mapping of each specimen, for a set of threshold values allowing separating microcracks from larger fractures: 3, 5, 10, and $50 \mu \mathrm{m}$. The results are shown on Fig. 6.

These results are displayed as total visible crack length in each aperture interval, per square meter of specimen surface, as a function of the progression of the mechanical loading process. This progression is quantified by $\epsilon / \epsilon_{\text {peak }}$, where $\epsilon_{\text {peak }}$ indicates the value of peak strain. The observation was limited to crack apertures exceeding $3 \mu \mathrm{m}$, as smaller strain values may be difficult to distinguish from the background noise of the mappings. Two observations can be made on the basis of these profiles:

- Smaller crack ranges always appear first during loading. The development of successive ranges of aperture follows the same order, from smaller to larger cracks. While this observation was to be expected, the clear visualisation of this succession indicates the validity of the methodology for quantifying cracks by ranges of size.

- In most samples, the smaller ranges of fissures reach a maximum around $\epsilon / \epsilon_{\text {peak }}=3$ to 4 , before decreasing. This phenomenon is caused by the presence of a macroscopic fracture, that initiates around $\epsilon=\epsilon_{\text {peak }}$. The opening of this fracture causes neighboring cracks to close, decreasing the total length of the smaller scales.

In addition to these results, a specific fracture area $A_{f r a c}$ was defined as such:

$$
A_{\text {frac }}(t)=\sum_{i} \bar{w}_{i} l_{i}(t)
$$




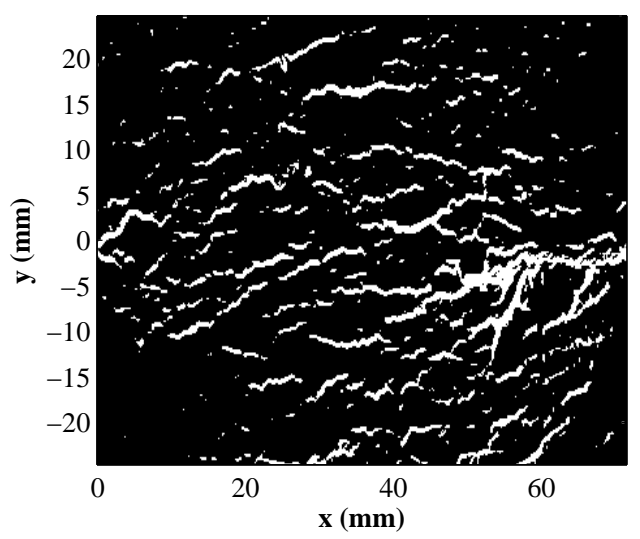

(a) $2 \mu \mathrm{m}$ threshold

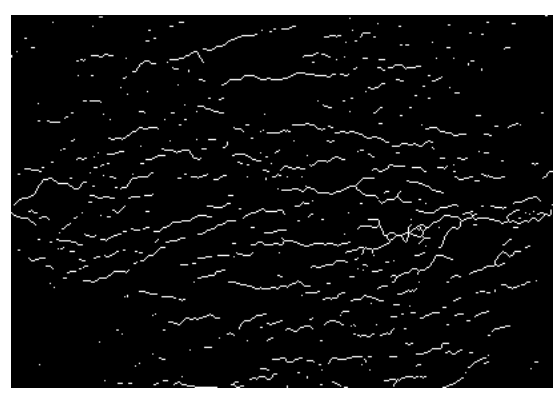

(c) $2 \mu \mathrm{m}$ threshold (skeletonised)

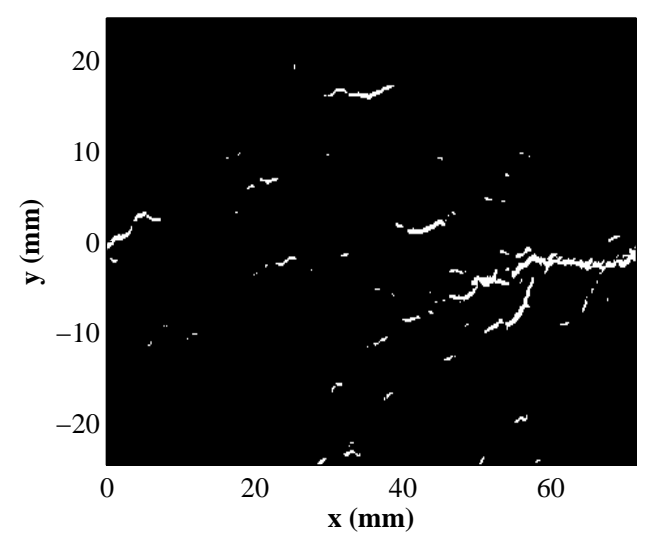

(b) $5 \mu \mathrm{m}$ threshold

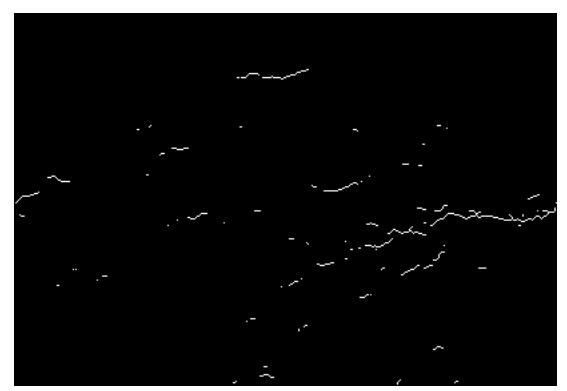

(d) $5 \mu \mathrm{m}$ threshold (skeletonised)

Figure 5: Image processing procedure for the measurement of crack size distributions: thresholds of $2 \mu \mathrm{m}$ (left) and $5 \mu \mathrm{m}$ (right) 


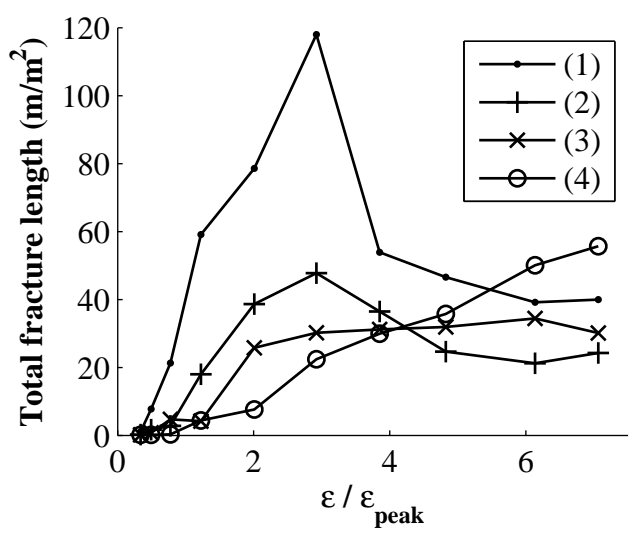

(a) Sample 1

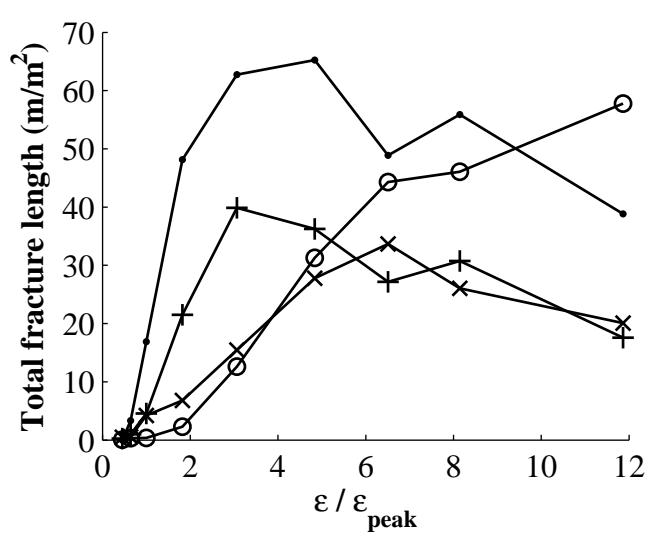

(c) Sample 3

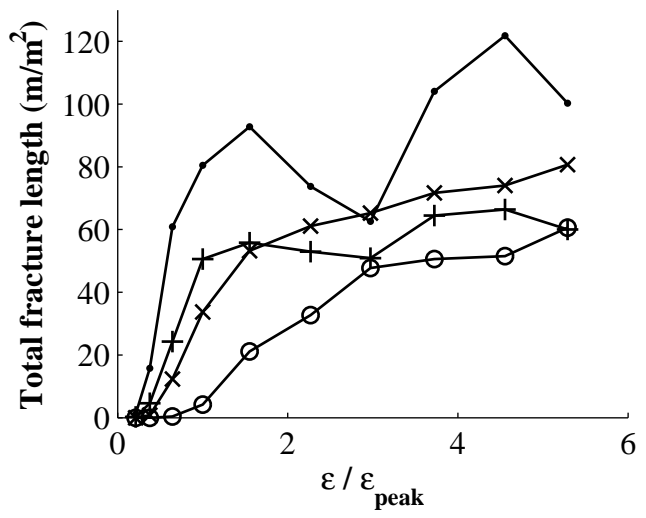

(e) Sample 5

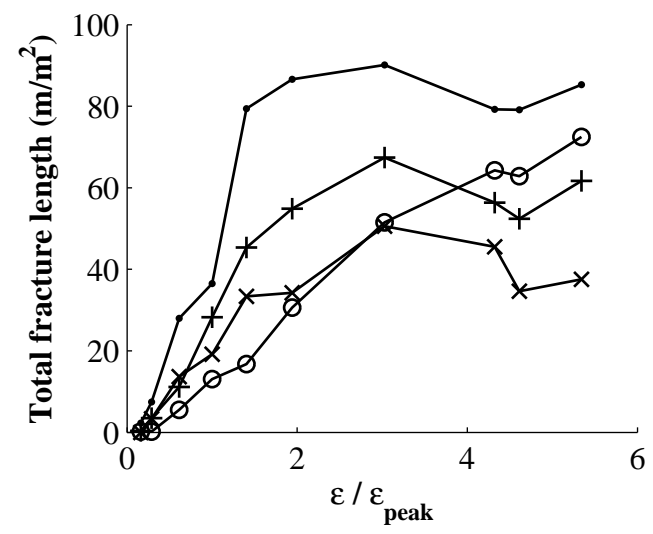

(b) Sample 2

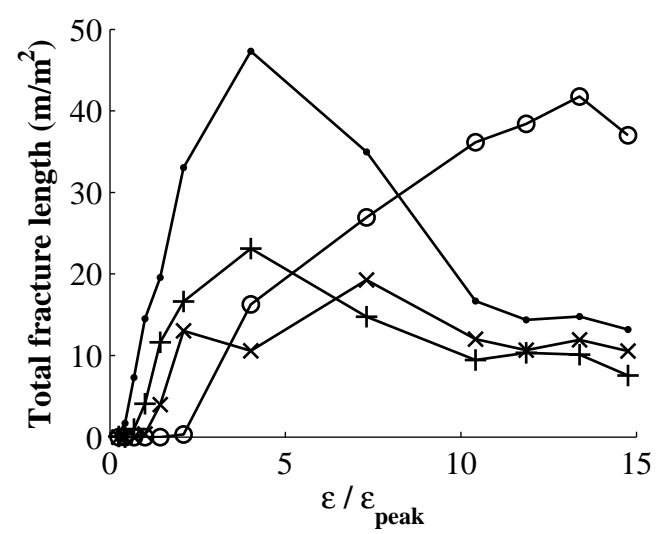

(d) Sample 4

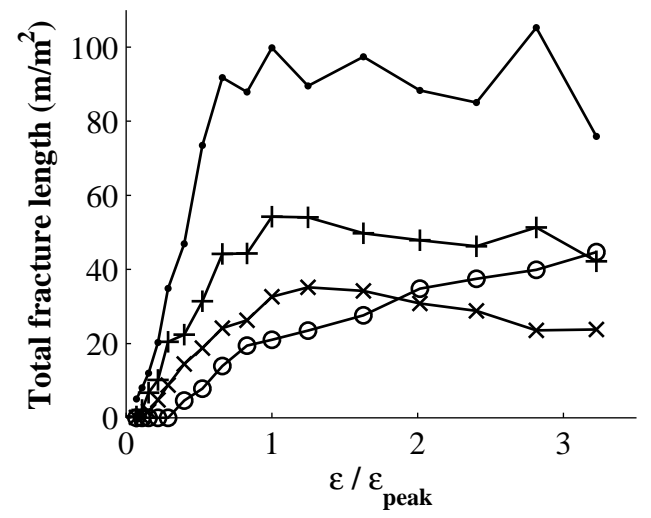

(f) Sample 6

Figure 6: Visible fracture length per square meter of sample surface : (1) $3 \leq w<5 \mu \mathrm{m}$, (2) $5 \leq w<10 \mu \mathrm{m}$, (3) $10 \leq w<50 \mu \mathrm{m}$, (4) $w \geq 50 \mu \mathrm{m}$ 
where the subscript $i$ ranges over the number of aperture subsystems measured by the image processing procedure. $\bar{w}_{i}$ is the mean crack width of the corresponding subsystem and $l_{i}$ its specific length, i.e. the ordinate of Fig. 6. The specific fracture area is therefore a summation of the visible lengths of all intervals, weighted by their respective openings. For the purpose of this calculation, the amount of aperture subsystems is greater than that displayed on Fig. 6: ranges were defined between values of 2, 3, 5, 10, 50 and $100 \mu \mathrm{m}$. $A_{\text {frac }}$ is non-dimensional and represents the ratio of visible sample surface occupied by cracks. The purpose of defining this quantity is the comparison of DIC and AE results, as explained below.

These measurements show that digital image correlation allows measuring all geometrical characteristics of cracks that may influence fluid flow in a construction material. With a relatively simple experimental setup, and the appropriate set of tools for image processing, the progressive opening and closing of discontinuities can be quantified without restriction of number or density. Although it is limited to 2-dimensional observations in the present case, the technique is therefore suitable to serve as a basis for the understanding of acoustic signals recordings. The following section shows how DIC can be used for the calibration of AE measurements, so that this technique can be autonomously used for damage monitoring of building components.

\section{Interpretation of acoustic emission}

\subsection{Damage quantification}

First, an attempt of global damage quantification was made on the basis of the total AE activity. This activity is represented by the number of localised $\mathrm{AE}$ events, i.e. signals received by three or more sensors. Noise effects are therefore expected to be reduced, as low-energy, non-localisable AE hits are not accounted for. Fig. 7 depicts the cumulative number of AE events and the loading profile of each sample.

Acoustic signals start emitting before the peak of loading at a slow rate. The recorded acoustic energy in this interval is low, and few signals can be captured as the material's acoustic attenuation is relatively high (approximately $0.25 \mathrm{~dB} \cdot \mathrm{mm}^{-1}$ ). These signals are emitted as a consequence of the previously observed microcracking. The profiles of cumulative AE events then increase significantly after peak strain: on all tests, high AE rates can be seen to occur simultaneously with sudden stress drops caused by crack propagation.

As a consequence of this observation, an attempt was made to correlate the total amount of visible fractures, previously observed by optical measurements, with the cumulated AE activity at each time of loading. For all available data points, the value of $A_{\text {frac }}$ was compared with the cumulated AE activity at the corresponding time of the test. Fig. 8 shows this comparison over all strain mappings captured during loading of all samples, representing a total of 83 data points.

It has already been found $[23,37]$ that the AE rate can give a quantitative indication on the damage level of construction materials. This is confirmed in the present case: the profile of fracture specific surface $A_{\text {frac }}$ versus cumulated AE events $N$ can be roughly approximated by a power law (see Fig. 8), resulting in a correlation coefficient of $R^{2}=0.9236$ between the results of both techniques (DIC and AE). Such an agreement is quite satisfactory, considering that the two techniques rely on very different physical principles. A connection was established between the amount of cracks appearing on the surface of the specimen and the rate at which elastic waves are released in the medium under tension. This observation confirms that $\mathrm{AE}$ can be used for damage quantification of building components with fair accuracy, once it has been calibrated by a method such as digital image correlation.

\subsection{Damage localisation}

The previous section addressed the matter of global damage quantification, and showed a connection between the cumulated number of AE events and the total opening of fractures. The resulting empiric evolution law, however, does not separate or identify cracks of different sizes, nor does it allow their localisation. The present section addresses the latter question by applying the localisation procedure, of which accuracy was previously assessed. In addition to the set of variables describing its waveform, each $\mathrm{AE}$ signal is assigned one temporal and two spatial coordinates. All localised sources were then superimposed with the strain mappings computed by the DIC algorithm. Fig. 9 shows the example of five successive stages of a sample, indicated on its loading profile 9(a). 


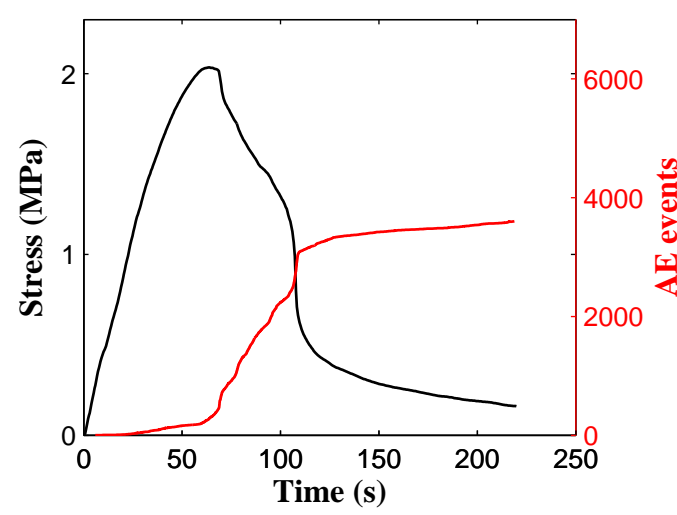

(a) Sample 1

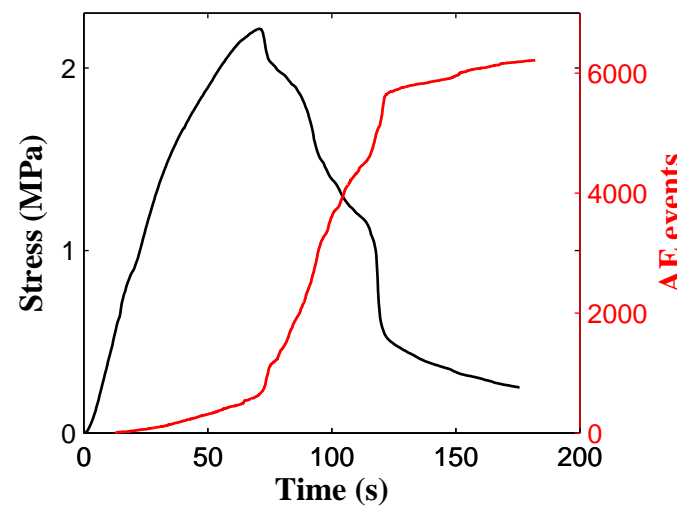

(c) Sample 3

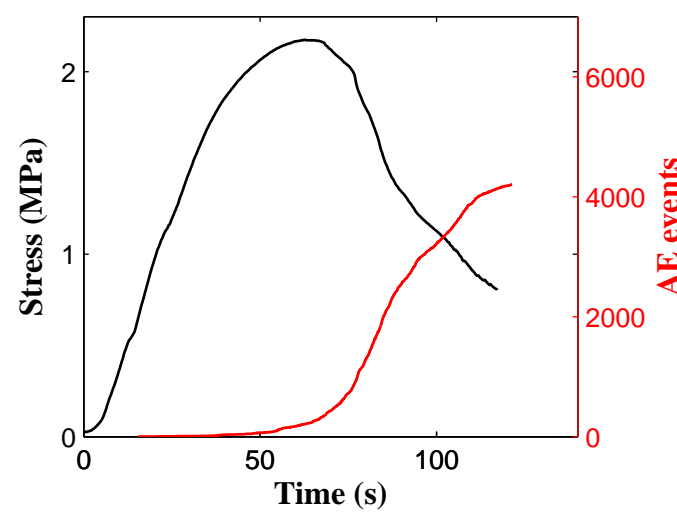

(e) Sample 5

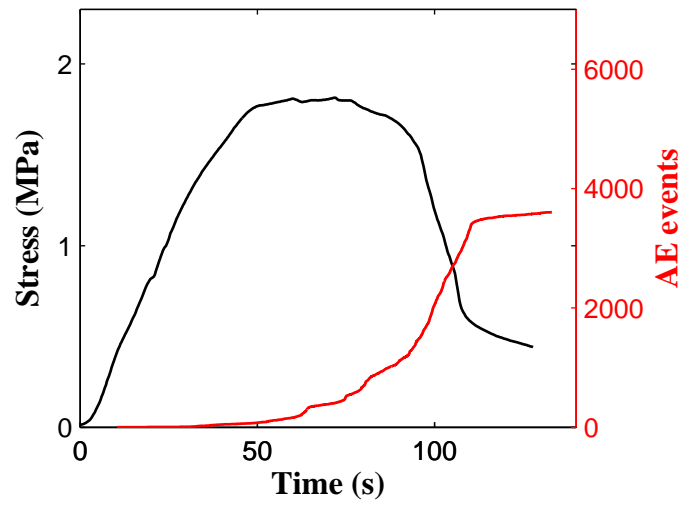

(b) Sample 2

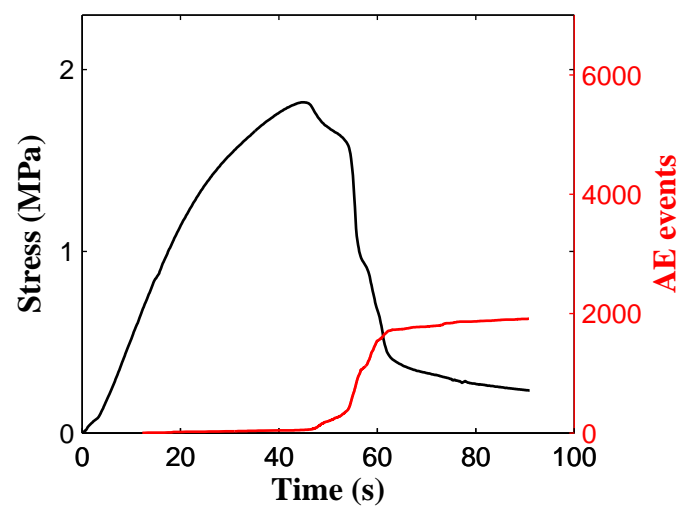

(d) Sample 4

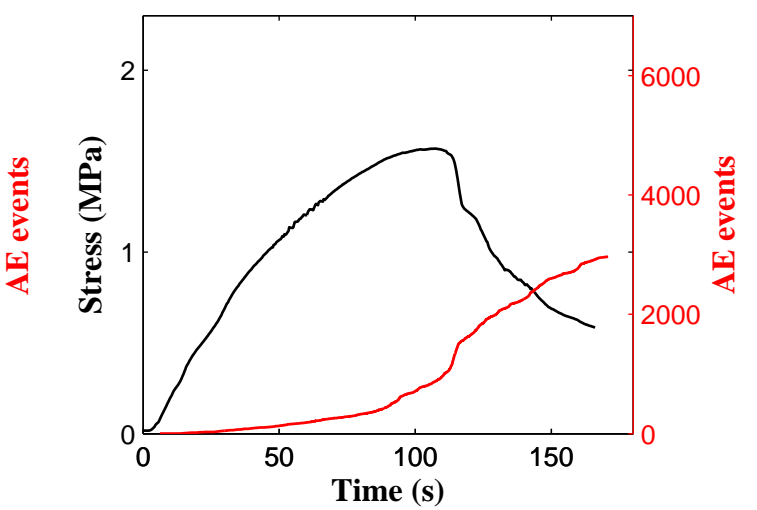

(f) Sample 6

Figure 7: Stress-strain profiles and cumulative AE signal distributions 


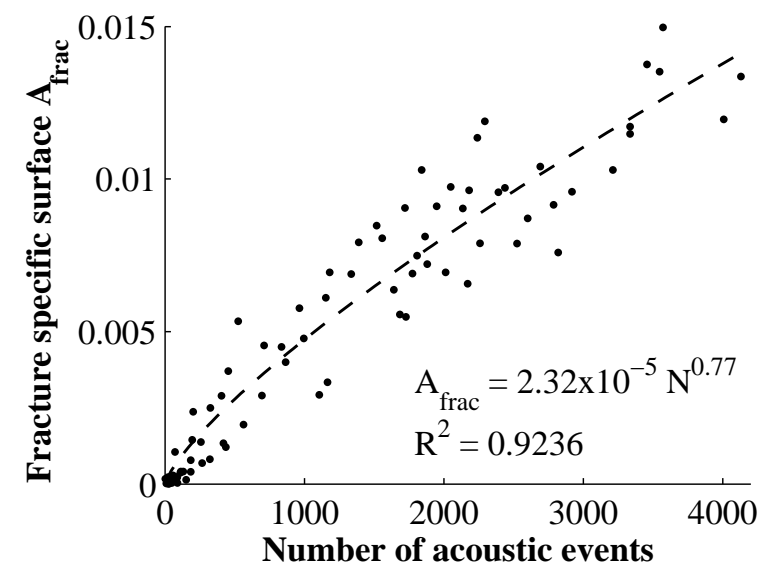

Figure 8: Fracture specific surface versus number of recorded acoustic events, and power law approximation

The first image (Fig. 9(b)) depicts the sample surface at the stress peak, at the time of initiation of a macroscopic crack. The red dots depict all AE sources that have been located from the beginning of the test. As can be seen on Fig. 7, the cumulative number of AE events is still low at this point. A moderate concentration of signals originates from a relatively large area around the fracture process zone, and occurs mostly ahead of the crack tip. After this point of the loading process, a higher emission rate is recorded during macroscopic crack expansion. Fig. 9(c) to 9 (f) show the state of the sample surface during its propagation. This propagation generates significantly higher concentrations of AE signals, that follow the crack trajectory.

The spatial correlation of both techniques is quite satisfying : most AE sources were located in areas of high strain, and their progress through the width of the sample is coordinated with the optically recorded crack propagation. The localisation procedure is however not flawless, as showed in the previous paragraph. This can explain the imperfect match between spots of high AE concentration and crack patterns. An other possibility is the fact that DIC only captures discontinuities that appear on the specimen surface, while AE sources originate from the entire volume. This matter is however minimised by the small thickness of the sample.

These measurements were carried out on all tested samples, and led to similar results. An important outcome of this procedure is the observation that $\mathrm{AE}$ signals seem to occur in two phases during the entire damage and fracture process. First, a moderate number of spatially scattered waves can be recorded as the material undergoes microcracking, distributed over a relatively large area. Then, during the propagation of a macroscopic crack, $\mathrm{AE}$ signals are captured at a much higher rate. Although the signal concentration is higher, their spatial distribution is still scattered, possibly because of the local variation of the AE wave propagation speed, which were not accounted for. Distributed signals continue to be emitted ahead of the crack tip. It must be recalled that most crack patterns appearing on the optical measurements have an aperture in the order of a few micrometers: AE monitoring allows the observation of crack far below the visible range.

\subsection{Fracture identification}

Signal localisation has added geometrical informations to the quantitative estimation of damage. In addition to informing on the global degradation state of the material, it has been shown that the AE technique may indicate where damage occurs, and give an estimate of the trajectory of the main cracks. However, it does not provide a complete understanding of damage mechanisms so far. The separation between microcracks and larger fractures has been graphically showed and translates into different concentrations of signals, but these observations do not allow their systematic separation.

$\mathrm{AE}$ amplitude is associated with the magnitude of the fracture, since the emitted energy depends on the crack opening displacement. Microcracks that develop before stress concentration and material failure are therefore 


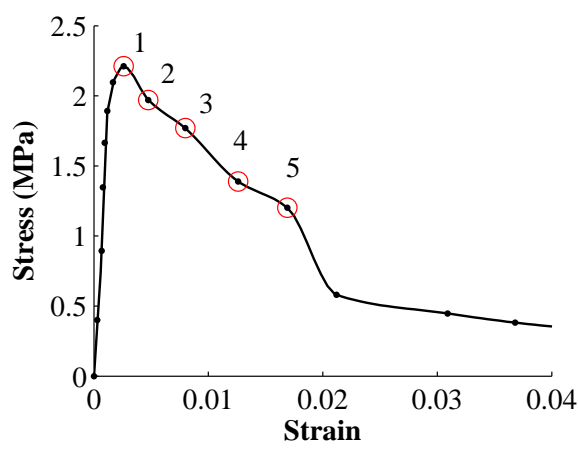

(a) Position of the images on the loading profile

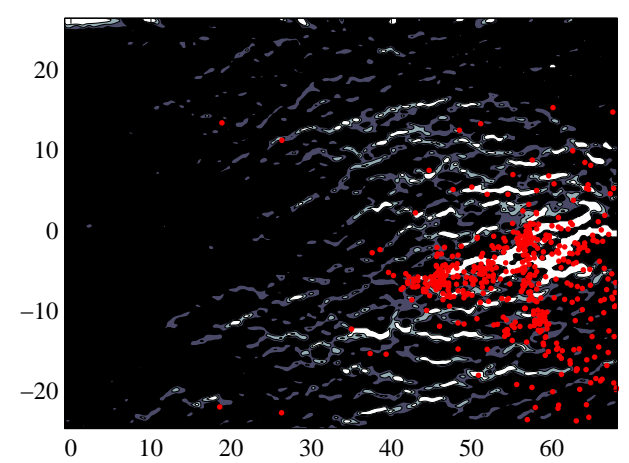

(c) Point 2

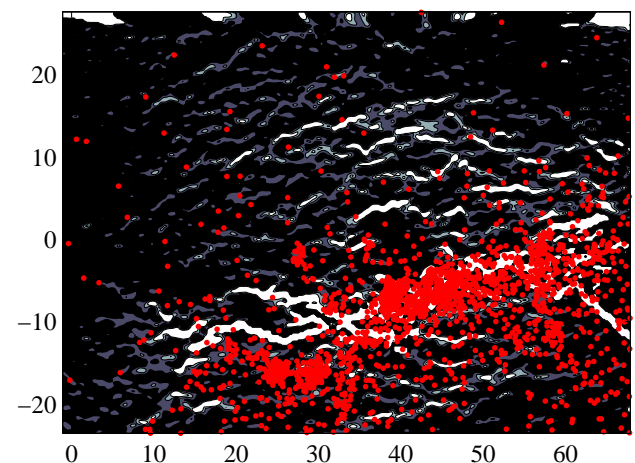

(e) Point 4

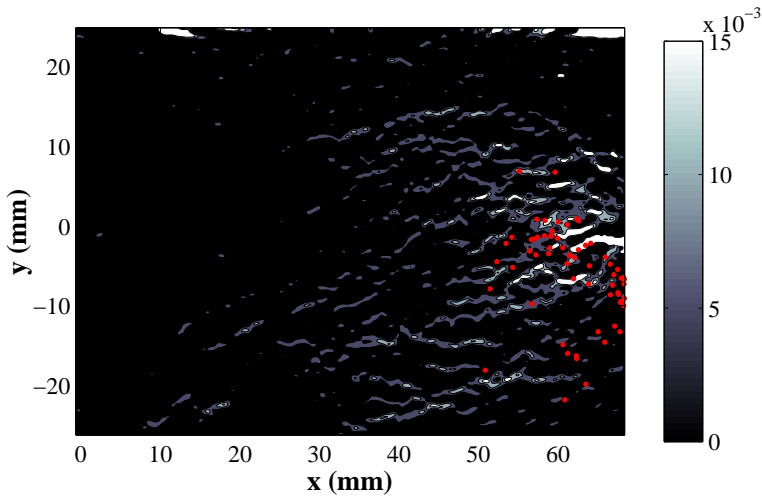

(b) Point 1

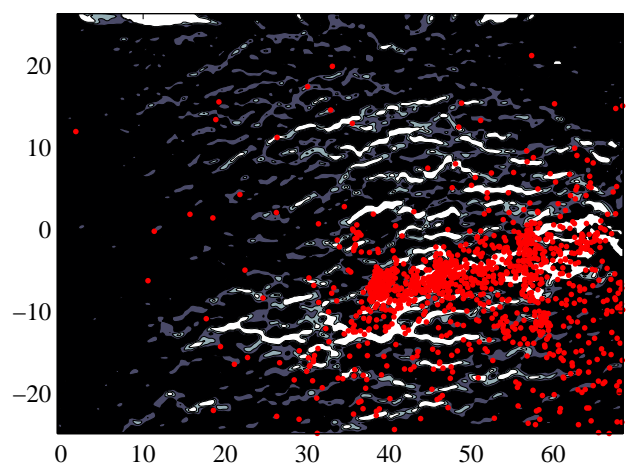

(d) Point 3

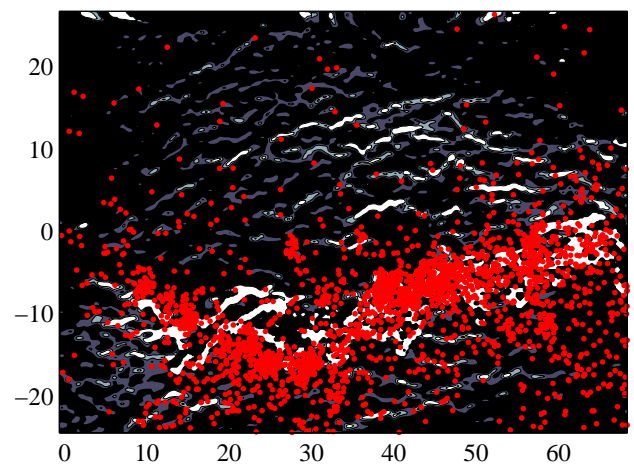

(f) Point 5

Figure 9: AE sources localisation (dots) and strain mapping (black and white patterns) 
expected to generate AE waves of smaller amplitude than larger fractures. This assumption can be made to study the development of the fracture process of concrete and rock-like materials.

In the field of seismology, the $b$-value serves as a predictive tool for material failure and is defined as the ratio of AE events of small to large amplitude:

$$
\log _{10} N=a-b A
$$

where $N$ is the number of signals, among a number of recently detected AE waves, exceeding a given amplitude $A$. At any time during the development of damage, the parameters $a$ and $b$ are defined by linear approximation of the current $N$ versus $A$ profile. High $b$-values therefore indicate a prevalence of microcracks, while lower values indicate their coalescence into macrocracks. An improved value of this indicator, or $I b$-value, was proposed by [31] and applied by [7, 1], among others, to study the fracture behaviour of concrete:

$$
I b=\frac{\log _{10} N\left(\mu-\alpha_{1} \sigma\right)-\log _{10} N\left(\mu+\alpha_{2} \sigma\right)}{\left(\alpha_{1}+\alpha_{2}\right) \sigma}
$$

where $\mu$ and $\sigma$ are the averaged value and standard deviation of the AE amplitude among the group of recent hits, $\alpha_{1}$ and $\alpha_{2}$ are filtering parameters (both were set to 0.5 in the present study). At any given time, $N\left(\mu-\alpha_{1} \sigma\right)$ indicates the number of recent events, of which amplitude exceeds $\mu-\alpha_{1} \sigma$ (similarly, $N\left(\mu+\alpha_{2} \sigma\right)$ indicates the number of recent events of which amplitude exceeds $\left.\mu+\alpha_{2} \sigma\right)$. The definition of the $I b$-value is similar to that of the $b$-value, with an additional filtering of non-representative signals of very high or low amplitude that might disturb the results.

At all times of a test, the $I b$-value is calculated from the amplitude distribution of the 200 latest consecutive signals. In order not to filter out waves of smaller amplitude, the procedure was applied on the basis of all recorded AE hits rather than of the localised events. Fig. 10 shows the $I b$ profiles superimposed with the loading curves of the fibre reinforced mortar samples.

As already mentioned, and seen by other authors [31,33], the evolution $I b$ is strongly related to the type of fracture. A common observation $[7,1]$ is that it starts at higher values as the first signs of damage are in the form of diffuse microcracking, generating mostly AE waves of low amplitude. Shortly before the initiation of a macroscopic fracture, these cracks aggregate: the energy release increases, decreasing the slope of the amplitude distribution. This results in a sudden drop of the $I b$-value shortly before the maximum stress, that acts as a warning for the specimen failure. Such a drop can be clearly seen on most samples of which the $I b$ profiles are shown on Fig. 10. In the case of the present material, a decrease of the value under 0.03 to 0.04 is a fair indicator for crack initiation. This trend is however not always clear: indeed, the material has a high acoustic attenuation and the number of low-energy signals, detected before macro-fracturing, may be low.

As can be seen on Fig. 9, the time of drop of the $I b$ indicator corresponds to the time at which AE sources start concentrating in a narrow band close to the point of macro-crack initiation. This observation indicates that, in addition to a quantitative estimation of damage, AE allows the separation of fissures according to their size, as well as the time and position of failure.

\section{Conclusion}

In the purpose of extending the use of non destructive damage observation to building materials, the present study consisted in the simultaneous use of digital image correlation and acoustic emission for the monitoring of progressive damage development in a formulation of fibre reinforced mortar under tensile loading. The aim was to assess the ability of AE to provide quantitative measurements of the crack networks, and to calibrate it with the support of an optical technique. In the course of the presented methodology, the following results were obtained:

- Digital image correlation, associated with an automated image processing procedure, allowed a precise twodimensional mapping of damage patterns, from micro-cracks to macroscopic fractures.

- The cumulative number of captured AE signals is a fair quantitative indicator of the total developed crack area. 


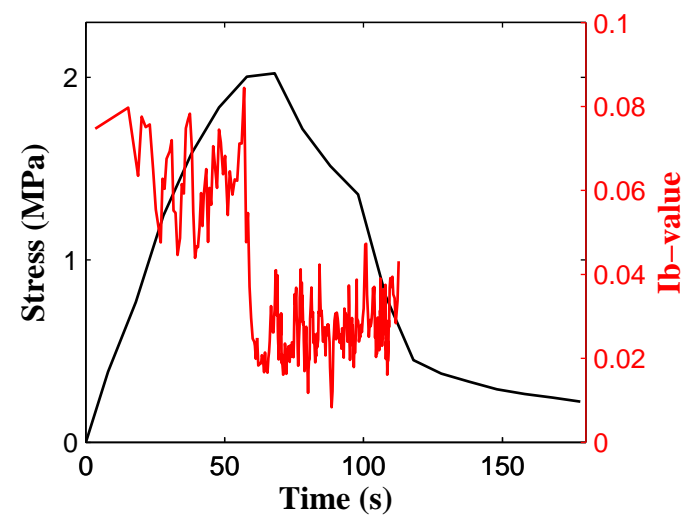

(a) Sample 1

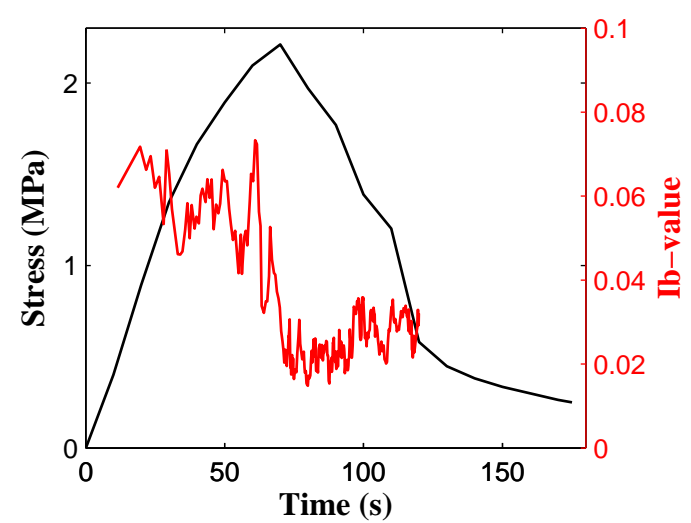

(c) Sample 3

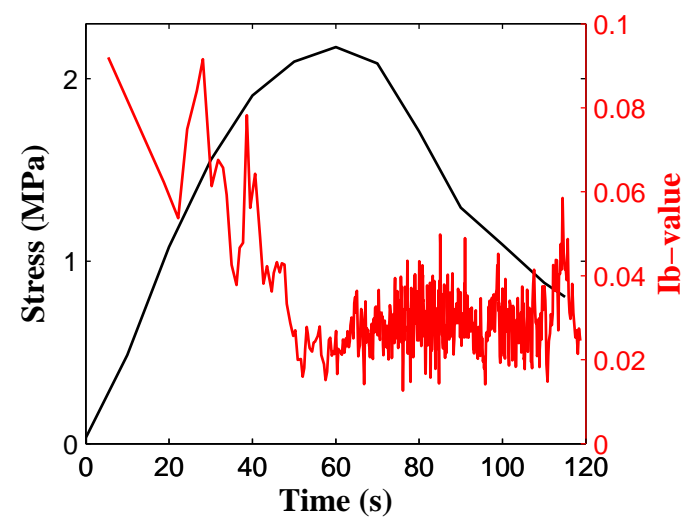

(e) Sample 5

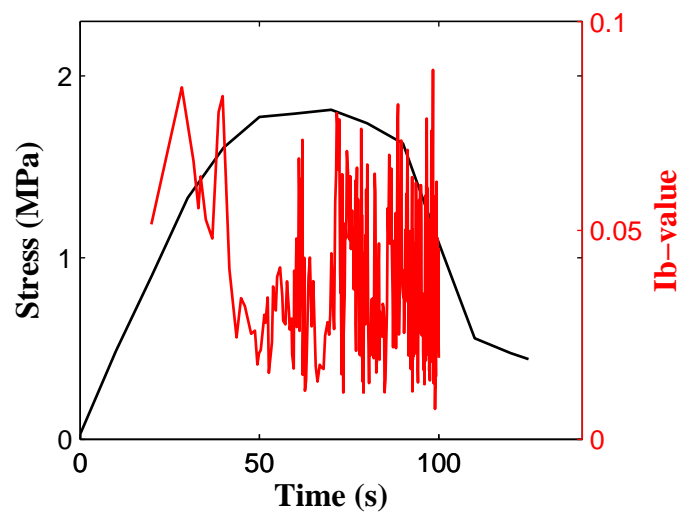

(b) Sample 2

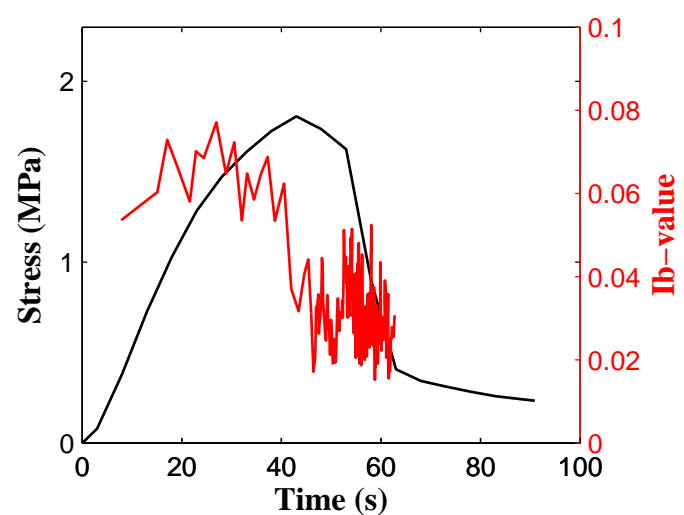

(d) Sample 4

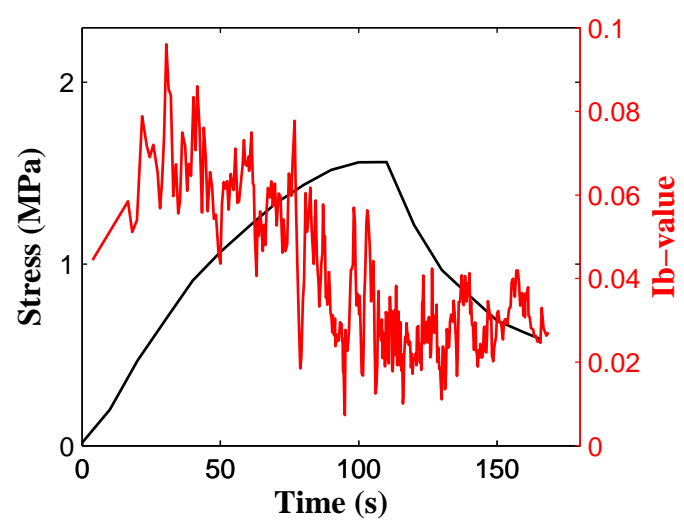

(f) Sample 6

Figure 10: Loading and $I b$-value profiles of fibre reinforced mortar 
- Before propagation of a macroscopic fracture, a moderate number of AE signals are emitted in a large area ahead of the crack tip. High concentrations of AE signals are then emitted along the crack trajectory, in fair accordance with damage mappings performed by digital image correlation.

- The time and position of initiation of a macro-crack can be anticipated: analysing the amplitude distribution of received signals allows visualising the transition between a regime of micro-cracks and the apparition of a larger fracture emitting waves of higher amplitude.

The methodology can however be improved: a better localisation can be reached by accounting for the variations of the AE propagation speed in the damaged material. This is partially possible with preliminary measurements performed in a diffusely damaged medium, but may be more challenging after fracture initiation and a highly nonuniform distribution of cracks. Furthermore, a more thorough interpretation of the AE waveforms may provide a better interpretation of the signals and a better understanding of the degradation mechanisms of the material.

\section{Acknowledgements}

The results presented in this paper have been obtained during the Renovbat project for the study of flow in fractured building components. Funding is provided by the Energy research cluster of the Rhône-Alpes region, which the authors would like to thank for their financial support through the project.

\section{References}

[1] D.G. Aggelis, D.V. Soulioti, N. Sapouridis, N.M. Barkoula, A.S. Paipetis, and T.E. Matikas. Acoustic emission characterization of the fracture process in fibre reinforced concrete. Constr Build Mater, 25:4126-4131, 2011.

[2] A. Ammouche, D. Breysse, H. Hornain, O. Didry, and J. Marchand. A new image analysis technique for the quantitative assessment of microcracks in cement-based materials. Cement Concrete Res, 30:25-35, 2000.

[3] G. Besnard, F. Hild, and S. Roux. "Finite-Element" displacement fields analysis from digital images: application to Portevin-Le Châtelier bands. Exp Mech, 46:789-803, 2006.

[4] F. Chalencon. Etude des intéractions rhéologie, fissuration et microstructure pour le développement d'un outil de formulation : application aux mortiers poreux minces fibrés dédiés à l'ITE. PhD thesis, INSA Lyon, 2010.

[5] F. Chalencon, L. Orgéas, P.J.J. Dumont, G. Foray, J.-Y. Cavaillé, E. Maire, and S. Rolland du Roscoat. Lubricated compression and X-ray microtomography to analyse the rheology of a fibre-reinforced mortar. Rheol Acta, 49:221-235, 2010.

[6] S. Choi and S.P. Shah. Measurement of deformations on concrete subjected to compression using image correlation. Exp Mech, 37:307-313, 1997.

[7] S. Colombo, I. G. Main, and M. C. Forde. Assessing damage of reinforced concrete beam using "b-value" analysis of acoustic emission signals. J Mater Civil Eng, 15:280-286, 2003.

[8] D. Corr, M. Accardi, L. Graham-Brady, and S. Shah. Digital image correlation analysis of interfacial debonding properties and fracture behavior in concrete. Eng Fract Mech, 74:109-121, 2007.

[9] H. Elaqra, N. Godin, G. Peix, M. R’Mili, and G. Fantozzi. Damage evolution analysis in mortar, during compressive loading using acoustic emission and X-ray tomography: effects of the sand/cement ratio. Cement Concrete Res, 37:703-713, 2007.

[10] N. Godin, S. Huguet, and R. Gaertner. Influence of hydrolytic ageing on the acoustic emission signatures of damage mechanisms occuring during tensile tests on unidirectionnal glass/polyester composites: application of a Kohonen's map. Compos Struct, 72:79-85, 2006. 
[11] S. Granger, A. Loukili, G. Pijaudier-Cabot, and G. Chanvillard. Experimental characterization of the selfhealing of cracks in an ultra high performance cementitious material: Mechanical tests and acoustic emission analysis. Cement Concrete Res, 37:519-527, 2007.

[12] F. Hild and S. Roux. Digital image correlation: from displacement measurement to identification of elastic properties - a review. Strain, 42:69-80, 2006.

[13] H. Hornain, J. Marchand, A. Ammouche, J.P. Commène, and M. Moranville. Microscopic observation of cracks in concrete - a new sample preparation technique using dye impregnation. Cement Concrete Res, 26:573-583, 1996.

[14] Z. Jia and S.P. Shah. Two-dimensional electronic speckle pattern interferometry and concrete fracture processes. Exp Mech, 34:262-270, 1994.

[15] E.N. Landis, E.N. Nagy, and D.T. Keane. Microstructure and fracture in three dimensions. Eng Fract Mech, 70:911-925, 2003.

[16] E.N. Landis, T. Zhang, E.N. Nagy, G. Nagy, and W.R. Franklin. Cracking, damage and fracture in four dimensions. Mater Struct, 40:357-364, 2007.

[17] Paul Leplay, Julien Réthoré, Sylvain Meille, and Marie-Christine Baietto. Damage law identification of a quasi brittle ceramic from a bending test using Digital Image Correlation. J Eur Ceram Soc, 30:2715-2725, 2010.

[18] E. Maillet, N. Godin, M. R'Mili, P. Reynaud, J. Lamon, and G. Fantozzi. Analysis of acoustic emission energy release during static fatigue tests at intermediate temperatures on ceramic matrix composites: towards rupture time prediction. Compos Sci Technol, 72:1001-1007, 2012.

[19] S. Momon, N. Godin, P. Reynaud, M. R'Mili, and G. Fantozzi. Unsupervised and supervised classification of AE data collected during fatigue test on CMC at high temperature. Compos Part A-Appl S, 43:254-260, 2012.

[20] S. Momon, M. Moevus, N. Godin, M. R'Mili, P. Reynaud, G. Fantozzi, and G. Fayolle. Acoustic emission and lifetime prediction during static fatigue tests on ceramic-matrix composite at high temperature under air. Compos Part A-Appl S, 41:913-918, 2010.

[21] S. Muralidhara, B.K. Raghu Prasad, Hamid Eskandari, and B.L. Karihaloo. Fracture process zone size and true fracture energy of concrete using acoustic emission. Constr Build Mater, 24:479-486, 2010.

[22] Kentaro Ohno and Masayasu Ohtsu. Crack classification in concrete based on acoustic emission. Constr Build Mater, 24:2339-2346, 2010.

[23] Masayasu Ohtsu and Hiroshi Watanabe. Quantitative damage estimation of concrete by acoustic emission. Constr Build Mater, 15:217-224, 2001.

[24] J.-J. Orteu. 3-D computer vision in experimental mechanics. Opt Laser Eng, 47:282-291, 2009.

[25] T.P. Philippidis, V.N. Nikolaidis, and A.A. Anastassopoulos. Damage characterization of carbon/carbon laminates using neural network techniques on AE signals. NDT\&E Int, 31:329-340, 1998.

[26] J. Réthoré, A. Gravouil, F. Morestin, and A. Combescure. Estimation of mixed-mode stress intensity factors using digital image correlation and an interaction integral. Int J Fracture, 132:65-79, 2005.

[27] S. Rouchier, G. Foray, M. Woloszyn, and J.-J. Roux. Influence of diffuse damage on the water vapour permeability of fibre-reinforced mortar. Transport Porous Med, 93:543-559, 2012.

[28] Simon Rouchier, Hans Janssen, Carsten Rode, Monika Woloszyn, Geneviève Foray, and Jean-Jacques Roux. Characterization of fracture patterns and hygric properties for moisture flow modelling in cracked concrete. Constr Build Mater, 34:54-62, 2012. 
[29] S. Roux and F. Hild. Stress intensity factor measurements from digital image correlation: post-processing and integrated approaches. Int J Fracture, 140:141-157, 2006.

[30] S.P. Shah and S. Choi. Nondestructive techniques for studying fracture processes in concrete. Int J Fracture, 98:351-359, 1999.

[31] T. Shiotani, K. Fujii, T. Aoki, and K. Amou. Evaluation of progressive failure using AE sources and improved B-value on slope model tests. Prog Acoust Emiss, 7:509-514, 1994.

[32] T. Shiotani, S. Momoki, H. Chai, and D.G. Aggelis. Elastic wave validation of large concrete structures repaired by means of cement grouting. Constr Build Mater, 23:2647-2652, 2009.

[33] Tomoki Shiotani, Masayasu Ohtsu, and Kenji Ikeda. Detection and evaluation of AE waves due to rock deformation. Constr Build Mater, 15:235-246, 2001.

[34] F.O. Slate and S. Olsefski. X-rays for study of internal structure and microcracking of concrete. JAm Concrete I, 60:575-588, 1963.

[35] M.A. Sutton, J.-J. Orteu, and H.W. Schreier. Image correlation for shape, motion and deformation measurements: basic concepts, theory and applications. Springer, 2009.

[36] M.A. Sutton, W.J. Wolters, W.H. Peters, W.F. Ranson, and S.R. McNeill. Determination of displacements using an improved digital correlation method. Image Vision Comput, 1:133-139, 1983.

[37] Tetsuya Suzuki and Masayasu Ohtsu. Quantitative damage evaluation of structural concrete by a compression test based on AE rate process analysis. Constr Build Mater, 18:197-202, 2004. 